\title{
DERIVAÇÃO DE UMA ESCALA DE TEMPO DE DESCORRELAÇÃO LAGRANGIANA PARA UMA CAMADA LIMITE ESTÁVEL
}

\author{
Virnei Silva Moreira ${ }^{2,}$ Gervásio Annes Degrazia ${ }^{1, *}$, Débora Regina Roberti, \\ Silvana Maldaner ${ }^{1}$ \\ ${ }^{1}$ Departamento de Física, Universidade Federal de Santa Maria, RS-Brasil \\ ${ }^{2}$ Universidade Federal do Pampa/UNIPAMPA, Itaqui, RS, Brasil \\ *gervasiodegrazia@gmail.com
}

\begin{abstract}
In the present study a new formulation for the lagrangian decorrelation time scale in the stable boundary layer (CBL) is proposed. This new model is compared with a well-known relation proposed by Sorbjan(1989).
\end{abstract}

\section{INTRODUÇÃO}

Uma quantidade fundamental para caracterizar a dispersão de contaminantes na Camada limite estável (CLE) é a escala de tempo de descorrelação Lagrangiana. Esta escala representa o efeito de memória associado aos padrões de turbulência existentes em uma Camada Limite Planetária (Degrazia et al., 2012). Desta forma, o desempenho dos modelos de dispersão depende diretamente de uma parametrização adequada desta escala de tempo. Assim, o objetivo deste trabalho é propor uma nova parametrização para a escala de tempo descorrelação lagrangiana para uma CLE. Esta escala de tempo é determinada a partir de estudos anteriores de Degrazia et al. (2000) e comparada com a parametrização proposta por Sorbjan (1989).

Uma escala de tempo de descorrelação lagrangiana, para todas as condições de estabilidade, foi derivada por Degrazia et al. (2000): 


$$
T_{L}=\frac{z}{\sqrt{c_{i}}}\left\{\frac{0,14\left[\left(-\overline{L / z} z_{i}\right)_{i} / L\right]^{1 / 2}}{\left[\left(f m^{*}\right)^{n+s}\right]^{2 / 3} w_{*}\left(\psi_{\varepsilon}^{c} z / z_{i}\right)^{1 / 3}}+\frac{0.059}{\left.\left(f m^{*}\right)_{i}^{c}\right]^{2 / 3}\left(\phi^{n+s}\right)^{2 / 3} u_{*}}\right\}
$$

Na expressão proposta por Degrazia et al.(2000) é necessário indicar expressões para $w_{*}, u_{*}, \phi_{\varepsilon}^{n+s},\left(f m^{*}\right)_{i}^{c},\left(f m^{*}\right)_{i}^{n+s}$ Para uma CLE estes parâmetros podem ser definidos de acordo com o apresentado na Tabela 1.

Tabela 1. Parâmetros para a camada limite estável

\begin{tabular}{|c|c|c|}
\hline$\phi_{\varepsilon}^{n+s}$ & $\phi_{\varepsilon}^{n+s}=\phi^{n}(1+3.7 z / \Lambda)$ & $\begin{array}{c}\text { Sorbjan } \\
(1989)\end{array}$ \\
\hline$\phi_{\varepsilon}^{n}$ & $\phi_{\varepsilon}^{n}=1.25$ & $\begin{array}{c}\text { Degrazia e } \\
\text { Moraes } \\
\end{array}$ \\
\hline$\Lambda$ & $\Lambda=L(1-z / h)^{\left(1.5 \alpha_{1}-\alpha_{2}\right)}$ & \\
& $\alpha_{1}=1.7$ & \\
& $\alpha_{2}=$ & \\
\hline$u_{*}$ & $u_{*}^{2}=\left(u_{*}^{2}\right)_{0}(1-z / h)^{\alpha_{1}}$ & Wyngaardet \\
& & al. (1974) \\
\hline
\end{tabular}

Substituindo-se as expressões apresentadas na Tabela 1 na Eq.(1) e utlizando-se a expressão geral para a variância turbulenta proposta por Degrazia et al. (2000), as seguintes expressões para a escala de tempo podem ser obtidas:

$$
T_{L u}=\frac{0.83 z}{\left(1+3.7 \frac{z}{L\left(1-\frac{z}{h}\right)^{1.25}}\right)\left(u_{*}\right)\left(1-\frac{z}{h}\right)^{0.75}}
$$




$$
T_{L v}=\frac{0.31 z}{\left(1+3.7 \frac{z}{L\left(1-\frac{z}{h}\right)^{1.25}}\right)\left(u_{*}\right)\left(1-\frac{z}{h}\right)^{0.75}}
$$$$
T_{L w}=\frac{0.19 z}{\left(1+3.7 \frac{z}{L\left(1-\frac{z}{h}\right)^{1.25}}\right)\left(u_{*}\right)\left(1-\frac{z}{h}\right)^{0.75}}
$$

\section{Resultados e conclusões}

Neste trabalho, uma escala de tempo para uma CLE baseada em argumentos de similaridade foi obtida. As expressões encontradas para $\mathrm{TL}_{\mathrm{u}, \mathrm{v}}$ são comparadas graficamente com as expressões propostas por Sorbjan (1989), Figura 1.

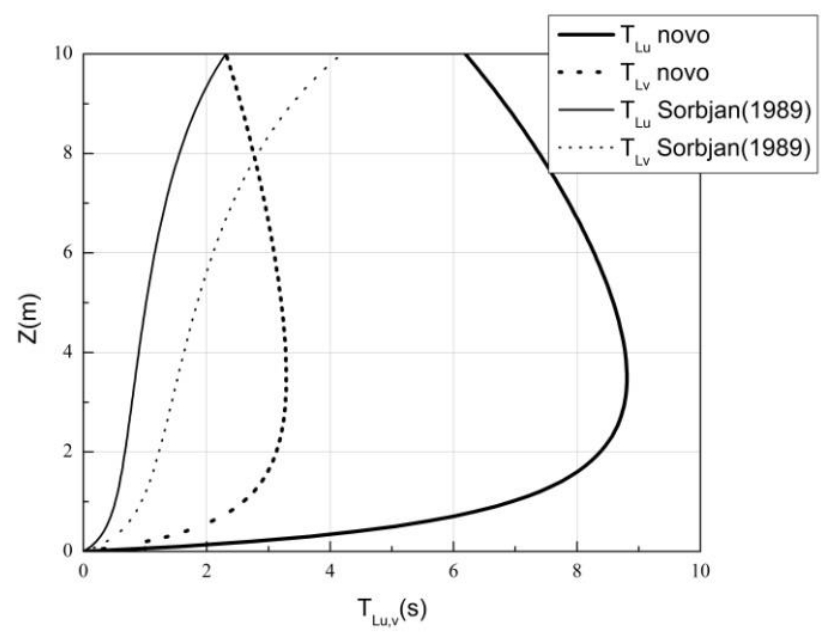

Figura 1. Comparação dos $T_{L u, v}$.

Observa-se que a nova expressão possui magnitude muito maior do que a proposta inicialmente por Sorbjan(1989). Assim, como trabalho futuro pretende-se empregar tais expressões em um modelo de dispersão lagrangiano. 


\section{AGRADECIMENTOS}

Os autores agradecem a CAPES, ao CNPq e a FAPERGS.

\section{REFERÊNCIAS}

SORBJAN, Z, 1989. Structure of the atmospheric boundary layer.Prentice Hall, 316 pp.

DEGRAZIA, G.A., MORAES, O.L.L. A model for eddy diffusivity in a stable boundary layer. Boundary-Layer Meteorology 58, 205-214,1992.

WYNGAARD, J.C., COTE, O.R., RAO, K.S., 1974. Modelling of the atmospheric boundary layer. Advances in Geophysics, V.18A. Academic Press, New York, pp. 193-212, 1974. 